\title{
Systems Theory: An Approach to Mass-Damper-Spring and Mass-Nondamper-Spring Systems
}

\author{
J. Sunday \\ Department of Mathematics, Adamawa State University, Mubi, Nigeria
}

\begin{abstract}
The concept of systems theory has been applied in various disciplines to analyze systems in such disciplines. In this research, systems theory was employed to model, analyze and study the natures of some problems in mass-spring systems. Mass-spring systems are second order linear differential equations that have variety of applications in science and engineering. They are the simplest model for mechanical vibration analysis. From the results obtained, it is clear that one of the systems was mass-damper-spring while the other was mass-nondamper-spring.
\end{abstract}

Keywords: Mass-damper-spring, mass-nondamper-spring, model, systems, systems theory

2000 MSC No: 65L05, 65LO6, 65D30

\section{Introduction}

Systems theory is an interdisciplinary field that studies the nature of systems from simple to complex-in society, cognition and science itself. In most models encountered in applications, the behavior is described through equations. The behavior, a subset of a universum, is simply defined as those elements of this universum satisfying a set of equations, called behavioral equations. In dynamical systems, these behavioral equations often take the form of differential or integral equations.

In this research, mass-spring systems described by the scalar second-order differential equations of the form

$$
m \frac{d^{2} x}{d t^{2}}+a \frac{d x}{d t}+k x=F(t)
$$

shall be considered; where $a$ is the damping coefficient, $k$ the spring constant, $m$ the mass attached to the lower end of the spring and $F(t)$ is the externally impressed force. It is assumed that this equation describes the motion of a unit mass in a mass-damper-spring combination or massnondamper-spring combination.

The system (1) is said to be

- asymptotically stable if and only if $a>0$ and $k>0$

- stable if and only if $a \geq 0$ and $k>0$ or $a>0$ and $k \geq 0$

- unstable if and only if $a<0$ or $k<0$ or $a=k=0$

*Corresponding author: joshuasunday2000@yahoo.com, sunday578@adsu.edu.ng 
Systems can be found in nature, science, society, economic context, and within information systems. Thus, systems theory finds applications in organization and business management, $[1,2]$. According to the authors in $[1,2]$, major constituents of many organizations which include business and human endeavors, are also regarded as systems since they are bounded by actions that are interrelated.

According to the author in [3], systems theory can also be applied in theory of learning and management popular called Theory U. Theory U proposes that the quality of the results that we create in any kind of social system is a function of the quality of awareness, attention or consciousness that the participants in the system operate from. Theory $U$ has five core elements/stages: co-initiating, co-sensing, co-presencing, co-creating, and co-evolving.

- Co-initiating common intent: stop and listen to others and to what life calls you to do,

- Co-sensing the field of change: go to the places of most potential and listen with your mind and heart wide open,

- Co-presencing inspiration and common will: go to the threshold and allow the inner knowing to emerge,

- Co-creating strategic microcosms: prototype the new to explore the future by doing. It also involves building structures, and creating a prototype of the new future and

- Co-evolving through innovations: ecosystems that facilitate seeing and acting from the whole. This stage also involves the co-development of a larger system that connects people across boundaries by seeing and acting from the whole.

This five stage process, allows the leader of an organization to see, hear, and act, in a way that incorporates and affect all levels, [3].

The author in [4] also stressed how important system theory is in the healthcare, this is because healthcare is a system with various levels of complexities. It has to do with policy makers, decision makers, and groups of people in organizations, institutions, and agencies that mold the way health care services are delivered to the society. The Health Care system also involves different levels of care, from providing palliative/end-of-life care to providing services for the prevention of diseases. Multiple health professionals, such as nurses, social workers, physicians, pharmacists, occupational therapists, and many other types of professionals provide healthcare is provided by. Systems theory is also applied in policy practice as recorded by [5], in cybernetics [6, 7], among others.

Mass-spring systems are applied in suspension of heavy-duty trucks, motion of a spring that is subject to frictional force (in the case of horizontal spring) or a damping force (in the case where a vertical spring moves through a fluid). The damping force supplied by a shock absorber in a car or bicycle is a simple example. In this case, the damping force is assumed to be proportional to the velocity of the mass and it acts in the direction opposite to the motion. It is important to state that this law has been established, at least approximately by some physical experiment.

\section{Definition 1.1 [8]}

A system may be defined as an organized entity made up of parts that are interrelated and interdependent. It is important to state that a system is more than the sum of its parts (subsystems).

It may also be defined as a regularly interacting or interdependent group of items forming a unified whole.

Definition 1.2 [9]

A mathematical model is a pair $(U, B)$ with $U$ a set, called the universum. Its elements are called outcomes and $B$ a subset of $U$ called behavior.

Definition 1.3 [9]

Let $U$ be a universum, $E$ a set, and $f_{1}, f_{2}: U \rightarrow E$. The mathematical model $(U, B)$ with $B=\left\{u \in U \mid f_{1}(u)=f_{2}(u)\right\}$ is said to be described by behavioral equations and is denoted by $\left(U, E, f_{1}, f_{2}\right)$. The set $E$ is called the equating space. We also call $\left(U, E, f_{1}, f_{2}\right)$ a behavioral equation representation of $(U, B)$. 
Definition 1.4 [10]

Any influence that exists within or upon an oscillatory system capable of reducing, restricting or preventing its oscillations is referred to as damping.

Damping in physical systems is produced by processes that dissipate the energy stored in the oscillation, [11]. Examples of this scenario occur in absorption and scattering of light in optical oscillators, resistance in electronic oscillators, and viscous drag in mechanical systems. Damping not based on energy loss finds applications in other oscillating systems such as those that occur in biological systems. Damping of a system can assume any of the following forms:

- Over-damping: here, the system goes back (exponentially decays) to equilibrium without oscillating,

- Critical damping: in this case, the system goes back to equilibrium as quickly as possible without oscillating,

- Under-damping: in under-damping, the system oscillates (at reduced frequency compared to the undamped case) with the amplitude gradually decreasing to zero, and

- Un-damping: this is a situation where the system oscillates at its natural resonant frequency. A very distinctive feature of systems theories is that it develops simultaneously across various disciplines and that scholars working from a systems theory perspective build on the knowledge and concepts developed within other disciplines, [12]. As a result, today we have several kinds of systems perspectives. There are viable systems (from Viable Systems Approach), smart systems (from systems thinking), economic systems (from economics), service systems (from Service Science, Management, Engineering and Design), reticular systems (from network theories), conceptual systems (from psychology), living systems (from natural sciences), social systems (from sociology), institutional systems (from law), ecosystems (from ecology) and technological systems (from cybernetics). This plurality resulted to a rich research stream with interdisciplinary contributions, [12].

\section{The General Systems Theory (GST)}

Ludwig von Bertalanffy, a biologist, in 1930 initiated the concept of GST at the University of Chicago. Bertalanffy began the GST study in life sciences; this eventually developed into the modern field of ecology (the study of systems of nature). He believed that nothing could be understood by isolating merely one part of what plays a significant role in a system. In his book, "General Systems Theory: Foundation, Development and Applications", Bertalanffy indicates the major aims of GST. These include;

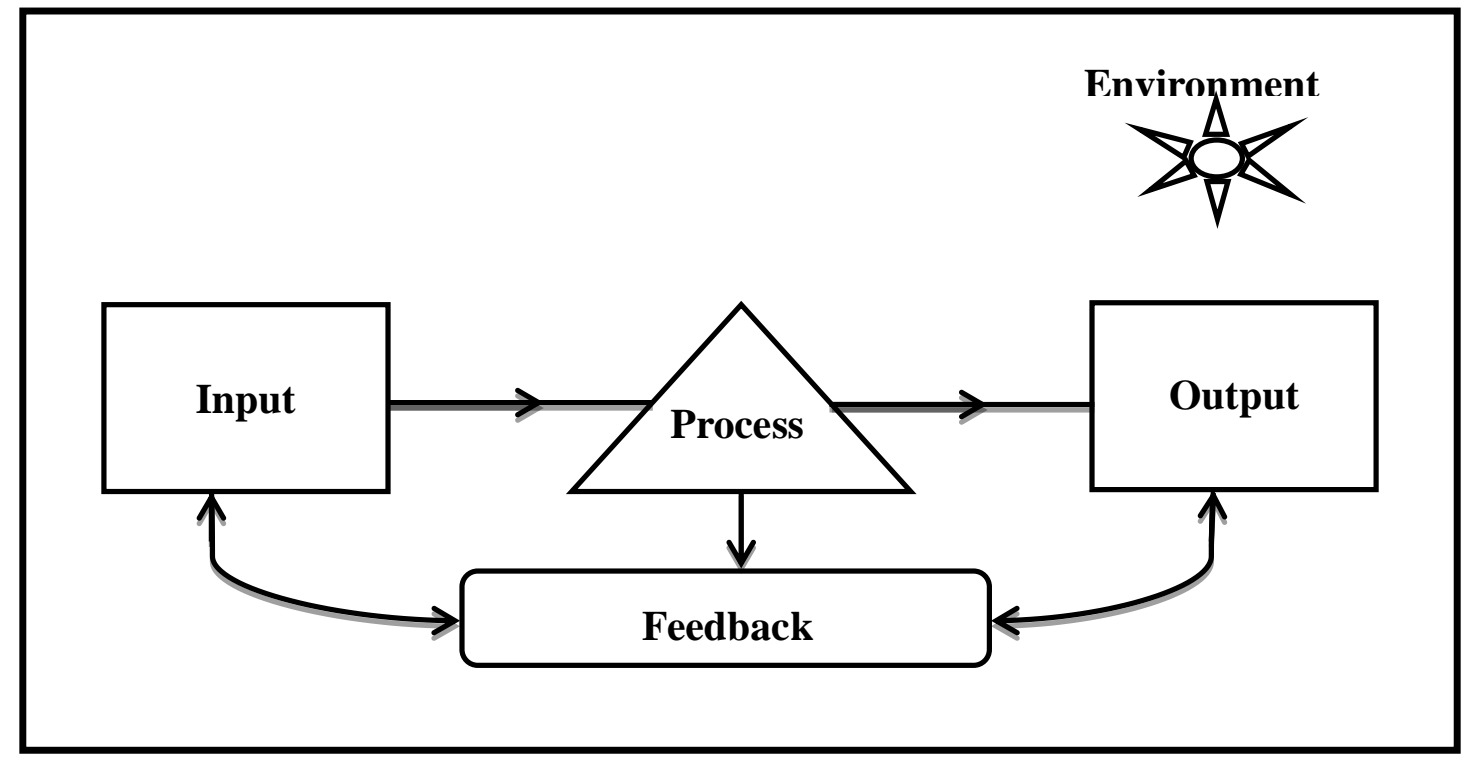

Figure 1: A general open system 
- The GST results in the integration of various sciences; both natural and social,

- A general theory of systems seem to be the focal point of such integration,

- Such theory serves as a means for aiming at exact theory in nonphysical fields of science,

- Such theory also brings us closer to the goal of the unity of science, and

- This result in a much-needed integration in scientific education, see [8].

According to him, if a system was going to be examined or understood, it had to be what he referred to as an open system. An open system is one in which the system has both inputs and outputs. To demonstrate this example, we can look to the human body as an open system. In order for life to be sustained, we must have oxygen, food and water to keep us alive. All of these components are what Bertalanffy labeled as inputs. However, he also explained that a system must have outputs or ways of excreting excess waste or unused portions from the input. If our bodies were to never release air or excrete waste, we would eventually self-implode because our body is not designed to simply keep receiving, see figure 1 above.

On the other hand, a closed system is one in which the system is self-sustaining and does not need input from outside sources; which means there is nothing to put out, [13]. The planet earth is a good example of a closed system, this is because the earth receives energy from the sun but it does not exchange mass with the solar system around it. Thus, scientists consider this idea of pure energy exchange without mass transference as a closed system. A true closed system is considered to be purely hypothetical or theoretical; this means that they can only exist in theory because every system needs some sort of input and output functions. However, on the opposite side, there are no perfectly open systems either. Open systems do have to have some point of restriction or closing in place to maintain balance through the self-regulating process. Bertalanffy's idea about systems theory is that nothing can be explained by isolating a component of system.

Some we state some important laws related to mass-spring systems.

Hooke's Law [10]: states that the restoring force $F$ exerted by a spring when it is elongated or compressed is proportional to the distance $e$ that it is elongated or compressed. That is, $F=k e$, where $k$ the constant of proportionality is referred to as the spring constant.

Newton's Second Law [10]: states that let $m$ be the mass of a body, $F$ its resultant force and $a$ be the acceleration produced in the body. Then, by Newton's second law, we have $F=m a$.

In the foregoing, the concept of systems theory shall be applied to model, analyze and determine the natures of some mass-spring systems.

\section{The Differential Equation Governing the Vibration of Mass-Spring Systems}

Let $l$ be the natural/unstretched length of a coil spring. Assuming a mass $m$ is attached to the lower end of the spring so that it comes to rest in its equilibrium position $O$. This elongates the spring by a quantity $e$, so that the elongated length is $l+e$. At the equilibrium position $O$, two forces act upon the mass $m$, i.e. the weight $m g$ acting vertically downwards and the spring force $k e$ acting vertically upwards (see Figure 2 below). Thus, we have

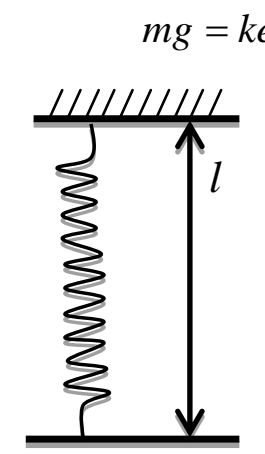

(a)

Figure 2: The mass-spring system

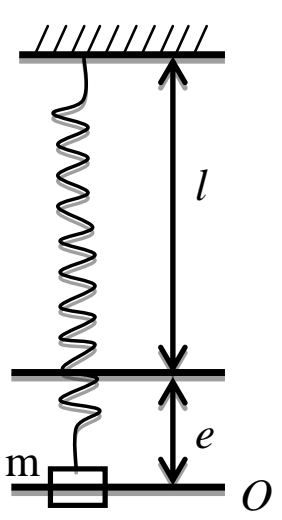

(b)

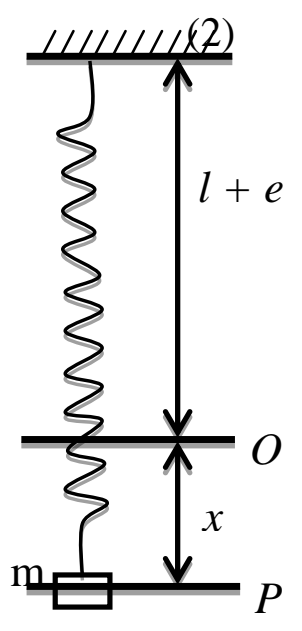

(c) 
Supposing $P$ is the position of the mass (below equilibrium position) at any time $t$, so that the distance from the equilibrium position $O$ to the point $P$ is given by $O P=x$. It is noteworthy that when the mass is below, at, or above its equilibrium position; then $x$ may be positive, zero or negative [10].

When the mass is positioned at $P$, it is acted upon by positive forces (i.e. the forces tending to pull the mass downward) and negative forces (i.e. the forces tending to pull the mass vertically upward).

(i) The weight $F_{1}=m g$, acting in the vertically downward direction

(ii) Let $F_{2}$ be the restoring force of the spring. When the mass is positioned at $P, F_{2}$ is negative because it acts in the upward direction. By Hooke's law, we have

$$
F_{2}=-k(x+e)
$$

Using (2) in (3), we get

$$
F_{2}=-k x-m g
$$

(iii) Let $F_{3}$ be the resisting force of the medium called damping force. For small velocities, it is clear that $F_{3}$ is proportional (approximately) to the magnitude of the velocity. When the mass moving downward (at $P$, say), $F_{3}$ acts in the upward direction (opposite to that of the motion) and so $F_{3}$ is negative and is given by,

$$
F_{3}=-a(d x / d t)
$$

(iv) External impressed force $F_{4}=F(t)$ acting in downward direction.

Therefore, by Newton's second law,

$$
F=m a
$$

where $F=F_{1}+F_{2}+F_{3}+F_{4}$ and $a=d^{2} x / d t^{2}$.

Thus,

$$
\begin{aligned}
& m\left(d^{2} x / d t^{2}\right)=m g-k x-m g-a(d x / d t)+F(t) \\
& m\left(d^{2} x / d t^{2}\right)+a(d x / d t)+k x=F(t)
\end{aligned}
$$

Equation (7) is the differential equation for the motion of a mass on a spring and is of the second order form (1).

If $a=0$, then we have a mass-nondamper-spring system otherwise the system is mass-damper-spring. If no external impressed forces exist, i.e. $F(t)=0$ for all $t$, the mass-spring system is called free, otherwise it is called forced, [10].

\section{Results}

In this section, the concept of systems theory shall be employed to model, analyze and determine the natures some mass-spring systems.

\section{Problem 4.1}

An $10 \mathrm{lb}$ weight is attached to the lower end of a coil spring that is suspended from the ceiling. The attached weight comes to rest at equilibrium position, thus stretching the spring 8 inch further. The attached weight is then pulled downwards 4 inch below its equilibrium position and then released at $t=0$ with an initial velocity of $1 \mathrm{ft} / \mathrm{sec}$ directed downwards. Assuming the resistance of the 
medium is neglected and that no external forces are present, formulate a differential equation modeling this mass-spring system problem. Take $g=32 \mathrm{ft} / \mathrm{s}^{2}$.

\section{Problem 4.2}

An $32 \mathrm{lb}$ weight is placed upon the lower end of a coil spring that is suspended from the ceiling. The weight comes to rest in its equilibrium position, thereby stretching the spring $2 \mathrm{ft}$. The weight is then pulled down 6 inch below its equilibrium position and released at $t=0$. Assuming no external forces are present, but the resistance of the medium is numerically equal to $4(d x / d t)$, where $d x / d t$ is the instantaneous velocity in feet per second. Formulate a differential equation modeling this mass-spring system problem. Take $g=32 \mathrm{ft} / \mathrm{s}^{2}$.

\section{Analysis (Problem 4.1)}

Let the natural length of the spring be $l$ inch. The mass $m=w / g=10 / 32=5 / 16$ slugs is attached to its lower end, thereby stretching the spring by an amount $e=8 \mathrm{inch}=2 / 3 \mathrm{ft}$ (note that $12 \mathrm{inch}=1 \mathrm{ft}$ ). In equilibrium position, the mass $m$ is acted upon by two forces:

(i) The weight $F_{1}=m g=10 \mathrm{lb}$ acting in the vertically downward direction

(ii) The restoring (spring) force $F_{2}=-k x-m g$ acting vertically upwards. Since $F=k e \Rightarrow 10=(2 / 3) k \Rightarrow k=15 \mathrm{lb} / \mathrm{ft}$, we obtain $F_{2}=-15 x-10$

On applying equation (6), we get

$$
F_{1}+F_{2}=m\left(\frac{d^{2} x}{d t^{2}}\right)
$$

which results to,

$$
\frac{d^{2} x}{d t^{2}}+48 x=0
$$

Thus, the system is a free mass-nondamper-spring system since $a=0$ and $F(t)=0$.

Analysis (Problem 4.2)

Let $e$ be the stretched length of the spring after the weight is attached. That is, $e=2 f t$. Also, $w=m g \Rightarrow 32=m \times 32$ so that $m=1$ slug

In the position of equilibrium, the mass $m$ is acted upon by three forces:

(i) The weight $F_{1}=m g=32 \mathrm{lb}$ acting in the vertically downward direction

(ii) The restoring (spring) force $F_{2}=-k x-m g$ acting vertically upwards. Since from Hooke's law $F=k e \Rightarrow 32=k \times 2 \Rightarrow k=16 f t$, we obtain $F_{2}=-16 x-32$

(iii) The resisting force $F_{3}$ of the medium is called the damping force. When the mass is moving downwards, $F_{3}$ acts in the upward direction (opposite to that of the motion) and so $F_{3}$ is negative, i.e.

$$
F_{3}=-4 \frac{d x}{d t}
$$

(iv) The external impressed force $F_{4}=F(t)$ is acting in downwards direction. In this case, $F_{4}=F(t)=0$ 
By Newton's second law,

$$
F=m a
$$

where

$$
a=\frac{d^{2} x}{d t^{2}}
$$

Thus,

$$
\begin{aligned}
& F_{1}+F_{2}+F_{3}+F_{4}=m\left(\frac{d^{2} x}{d t^{2}}\right) \\
& 32+(-16 x-32)-4 \frac{d x}{d t}+0=\frac{d^{2} x}{d t^{2}}
\end{aligned}
$$

which results to,

$$
\frac{d^{2} x}{d t^{2}}+4 \frac{d x}{d t}+16 x=0
$$

This system is a free mass-damper-spring system since $a \neq 0$ and $F(t)=0$.

\section{Conclusion}

Systems theory has been successfully applied to model, analyze and interpret the natures of massspring systems. It is clear from the results obtained that one of the systems is a mass-damper-spring system while the other is a mass-nondamper-spring system.

\section{References}

[1] F. E. Kast, and J. E.Rosenzweig, General systems theory: Applications for organization and management. Academy of Management Journal, 15(4), (1972):447-465.

[2] P.Sege, The fifth discipline: The art and practice of the learning organization ( ${ }^{\text {nd }}$ Edition), New York, (2006).

[3] O. Scharmer, Theory U. Leading form the future as it emerges. San Francisco, CA: BerretteKoehler Publishers, (2009).

[4] C. P. Cordon, System theories: An overview of various system theories and its application in healthcare. American Journal of Systems Sciences, 2(1), (2013):13-22. Doi: 10.5923/j.ajss.20130201.03.

[5] J. Stewart, and R. Ayres, Systems theory and policy practice: An exploration. Kluwer Academic Publishers, (2001). Doi.org/10.1023/A:1010334804878.

[6] G. M. Gonille, L'approccio sistemico al governo di impresa-Verso la scienticazione dell'azione di governo. Padova:CEDAM, (2008).

[7] S. Banile, Limpresa come sistema-Comtributi sull'Approccio sistemico Vitale , $2^{\text {nd }}$ Ed. Torino: Giappichelli, (2006).

[8] L. Bertalanffy, General systems theory. Foundations, development and applications. George Braziller Publishers, New York (1968).

[9] J. W. Polderman, and J. C. Willems, Introduction to the mathematical theory of systems and control, 1998. 
[10] M. D. Raisinghania, Ordinary and partial differential equations. S. Chand and Company PVT Ltd, New-Delhi 110 055, 17 $7^{\text {th }}$ Edition, (2014).

[11] J.Sunday, A. A.James , M. R. Odekunle, and A. O. Adesanya, Solutions to free undamped and free damped motion problems in mass-spring systems. American Journal of Computational and Applied Mathematics, 6(2), (2016):82-91. Doi:10.5923/j.ajcam.20160602.08.

[12] C. Mele, J. Pels, and F. Polese, (2010). A brief review of systems theories and their managerial applications. Institute for operations research and management, 2(1-2), (2010):126-135. Doi.org/10.1287/serv.2.1_2.126.

[13] A. Heil, Systems theory, SPC 330, 2017. 